\title{
Long-Term Survival Benefit of Coronary Revascularization in Patients Undergoing Stress Myocardial Perfusion Imaging
}

\author{
Mario Petretta, MD; Wanda Acampa, MD, PhD; Stefania Daniele, PhD; Emilia Zampella, MD; \\ Roberta Assante, MD; Carmela Nappi, MD; Marco Salvatore, MD; Alberto Cuocolo, MD
}

\begin{abstract}
Background: We assessed the relationship between clinical outcome and coronary revascularization according to stress-gated myocardial perfusion single-photon emission computed tomography (MPS) in an observational series of patients with suspected or known coronary artery disease (CAD), on long-term follow-up.

Methods and Results: The study group consisted of 2,059 patients. During a median follow-up of 61 months, 184 events occurred (126 cardiac deaths and 58 non-fatal MI). The impact of revascularization during follow-up on eventfree survival was evaluated using an extended Cox regression model, adjusting for potential clinical and MPS confounders. Revascularization was treated as a binary non-reversible time-dependent covariate. Predefined interactions tested were: (1) revascularization and summed difference score (SDS); (2) revascularization and post-stress left ventricular (LV) ejection fraction (EF); and (3) SDS and post-stress LVEF. Revascularization had a significant effect on event-free survival (adjusted HR, 0.19; $P<0.001$ ). Significant interactions were found between revascularization and SDS $(P=0.045)$, and between LVEF and SDS $(P=0.015)$. The protective effect of revascularization increased as SDS increased. For SDS $<6$ the reduction in HR was detectable only for reduced LVEF.
\end{abstract}

Conclusions: Both the degree of stress-induced ischemia and LVEF predict the effect of revascularization on outcome in patients with suspected or known CAD. The protective effect of revascularization appears to be greater in patients with severe ischemia and preserved LVEF. (Circ $J$ 2016; 80: 485-493)

Key Words: Coronary revascularization; Imaging; Prognosis

$\mathbf{T}$ he prognostic value of stress myocardial perfusion single-photon emission computed tomography (MPS) in patients with suspected or known coronary artery disease (CAD) has been extensively demonstrated. ${ }^{1}$ Given that coronary revascularization might change the natural history of $\mathrm{CAD}$, in prognostic studies patients undergoing revascularization are often censored at the time of the procedure. In contrast, revascularization may be associated with increased mortality in high-risk subsets. ${ }^{2}$ The role of stress MPS for identifying patients who may benefit from revascularization is an area of active research. ${ }^{3}$ Revascularization may reduce the absolute and relative risk of cardiac death more than medical therapy in patients with moderate-large amounts of stressinduced myocardial ischemia. ${ }^{4}$ Although left ventricular (LV) ejection fraction (EF) may also predict cardiac death, only inducible ischemia identifies which patients have short-term benefit from revascularization. ${ }^{5}$ We assessed, on long-term follow-up, the relationship between clinical outcome and coronary revascularization on stress-gated MPS in an obser- vational series of patients.

\section{Editorial p 325}

Methods
Subjects
A total of 2,157 consecutive patients with available rest and
stress-gated MPS data, referred for the detection of myocar-
dial ischemia between 2001 and 2010 , were selected from the
prospective data bank of the University of Naples Federico II
Hospital. As part of the baseline examination, information on
cardiovascular risk factors, presenting symptoms, and CAD
history were collected at the time of MPS. Patients were
excluded for (1) recurrent chest pain unresponsive to anti-
ischemic medications; (2) recent acute coronary syndrome
(ACS), stroke, or transient ischemic attack (past 3 months); (3)
uncompensated congestive heart failure or recent admission
for congestive heart failure; (4) significant valvular or

Received October 15, 2015; revised manuscript received November 13, 2015; accepted November 18, 2015; released online December 18, 2015 Time for primary review: 13 days

Department of Translational Medical Sciences (M.P.), Department of Advanced Biomedical Sciences (E.Z., R.A., C.N., A.C.), University of Naples Federico II, Naples; Institute of Biostructure and Bioimaging, National Council of Research, Naples (W.A., S.D.); and IRCCS SDN, Naples (M.S.), Italy

Mailing address: Alberto Cuocolo, MD, Department of Advanced Biomedical Sciences, University of Naples Federico II, Via Pansini 5, 80131 Naples, Italy. E-mail: cuocolo@unina.it

ISSN-1346-9843 doi:10.1253/circj.CJ-15-1093

All rights are reserved to the Japanese Circulation Society. For permissions, please e-mail: cj@j-circ.or.jp 
congenital heart disease; (5) absolute contraindication to dipyridamole in patients unable to exercise; and (6) significant comorbidity reducing life expectancy to $<1$ year. The institutional review committee approved the study and all patients gave informed consent.

\section{Gated MPS}

All patients underwent same-day $99 \mathrm{~m} \mathrm{Tc}$ sestamibi rest and stressgated MPS by exercise, or dipyridamole stress test according to the recommendations of the European Association of Nuclear Medicine and European Society of Cardiology, ${ }^{6}$ as previously described in detail. ${ }^{7}$ In all patients, $\beta$-blockers and calcium antagonists were withheld for $48 \mathrm{~h}$ and long-acting nitrates for $12 \mathrm{~h}$ before testing. Imaging was performed using a dual-head rotating gamma camera (E.CAM, Siemens Medical Systems, Hoffman Estates, IL, USA) equipped with a lowenergy, high-resolution collimator and connected to a dedicated computer system. ${ }^{8}$ No attenuation or scatter correction was used. For gating, a cardiac cycle was divided into 8 frames. The R-R interval and heart rate histogram were recorded to monitor arrhythmia. An average RR interval of $\pm 15 \%$ was accepted for gating. Perfusion imaging was reconstructed by summing the gated data at each projection into an ungated raw data file before low-phase pre-filtering and ramp-filtered back projection.

e-soft version 2.5 (QGS/QPS, Cedars-Sinai Medical Center, Los Angeles, CA, USA) was used to calculate LV volumes and $\mathrm{EF}$ and the scores incorporating both the extent and severity of perfusion defects, using standardized segmentation of 17 myocardial regions. ${ }^{9}$ Briefly, this commercial package determines reconstruction limits for the projection dataset, reconstructs the projection images into trans-axial images using standard filtered back projection, and then reorients the transaxial images into short-axis images. LV contours were checked visually and manually adjusted if the computer-generated automatic contours were found to be incorrect. Quantitative defect extent and severity were defined using sex-specific normal limits, and summed stress score (SSS) was obtained by adding the scores of the 17 segments ( 0 , normal; to 4 , absent perfusion) of the stress images. A similar procedure was applied to the resting images to calculate the summed rest score (SRS). The summed difference score (SDS) represents the difference between the stress and rest scores and is used as an index of ischemic burden: SDS 0-1, no ischemia; 2-6, mild ischemia; $>6$, moderate-severe ischemia. ${ }^{10}$ Post-stress LVEF $>45 \%$ and SSS $<3$ were considered normal. ${ }^{11,12}$

\section{Follow-up Data}

Patient follow-up was obtained via phone questionnaire to all patients and general practitioners or cardiologists and by review of hospital or physician records by individuals blinded to the test results. The outcome was a composite endpoint of cardiac death or non-fatal myocardial infarction (MI), whichever occurred first. The cause of death was confirmed on review of the death certificate, hospital chart, or physician records. Two physicians (R.A., E.Z.) reviewed each death, rescreened medical records when appropriate, and resolved disparity by consensus. Death was considered to be of cardiac origin if the primary cause was defined as acute MI, congestive heart failure, valvular heart disease, sudden cardiac death, or cardiac interventional/surgical procedure related. Sudden cardiac death was defined as either a documented arrhythmogenic death or out-of-hospital occurrence of an unexpected presumed pulseless condition together with the absence of an obvious non-cardiac explanation. MI was defined as $\geq 2$ of the following 3 criteria: chest pain or equivalent symptom complex; positive cardiac biomarkers; or typical electrocardiographic changes. ${ }^{13}$ Patients with non-cardiac death during follow-up were censored at the time of death. Revascularization procedures during follow-up, and time to first revascularization from index MPS were noted. The decision on revascularization was made by the referring physician, taking into account all available information and patient preferences. The date of the last examination or consultation was used to determine the length of follow-up.

\section{Statistical Analysis}

Continuous variables are expressed as mean \pm SD and categorical data as percentage. Student's t-test, chi-squared test, or Fisher exact test were used as appropriate. For continuous data, differences among groups were compared using analysis of variance. If a significant F-value was found, post-hoc analysis with Bonferroni correction was performed. Correlation between continuous variables was estimated with Pearson's correlation coefficient and multivariate linear regression analysis. Two-tailed $\mathrm{P}<0.05$ was considered statistically significant. Because patients shifted from the no revascularization to the revascularization group during follow-up, unadjusted eventfree survival functions were non-parametrically estimated using the Simon-Makuch method, ${ }^{14}$ a modification of KaplanMeier analysis, and compared using the Mantel-Byar test. ${ }^{15}$ In this context, revascularization is treated as a binary nonreversible time-dependent covariate that starts at 0 (no revascularization) and may switch to 1 (revascularization) at some time point. Thus, the study cohorts are continually updated as follows: for the time prior to revascularization, the patient contributes to the no revascularization cohort, but for the time after undergoing revascularization, the patient contributes to the revascularization cohort. Thus, the start time ( $\left.t_{0}\right)$ for the no revascularization group is the beginning of follow-up, while for the revascularization group to is the date of revascularization after index MPS.

The impact of coronary revascularization on event-free survival was evaluated with the extended Cox regression model. To take into account differences in the time from index MPS to revascularization, revascularization was treated as a binary non-reversible time-dependent covariate. Thus, revascularized patients were considered to be members of the no revascularization group until the day of intervention. At that point, they were crossed over to the revascularization group. This means that patients contribute to the hazard estimate of the no revascularization group up to the revascularization time, when they start to contribute to the hazard estimate of the revascularization group. The extended Cox model was run with and without adjusting for patient age, gender, diabetes, hypertension, dyslipidemia, smoking, family history of CAD, chest pain symptoms, history of MI, stress test type, poststress LVEF, SSS, SRS and SDS. Predefined interactions were tested using the partial likelihood ratio test. The interactions tested were: (1) coronary revascularization and SDS; (2) coronary revascularization and post-stress LVEF; and (3) SDS and post-stress LVEF. HR and 95\% CI are reported. To simplify interpretation of the parameter estimates, age and post-stress LVEF were centered at 40 years and $45 \%$, respectively. For all covariates, except revascularization, the proportional hazard assumption was validated by visual inspection of the $\log [-\log$ (survivor function) $]$ for categorical variables and with statistical tests based on Schoenfeld residuals for continuous variables. The linearity of the relationship between covariates and event-free survival in the Cox model was assessed using 


\begin{tabular}{lcccc|}
\hline Table 1. Subject Characteristics vs. Cardiac Death or Non-Fatal MI & & \\
& $\begin{array}{c}\text { All patients } \\
(\mathbf{n = 2 , 0 5 9 )}\end{array}$ & $\begin{array}{c}\text { Events } \\
(\mathbf{n = 1 8 4 )}\end{array}$ & $\begin{array}{c}\text { No events } \\
(\mathbf{n = 1 , 8 7 5 )}\end{array}$ & P-value \\
Age (years) & $61.0 \pm 10.4$ & $65.8 \pm 10.5$ & $60.5 \pm 10.3$ & $<0.001$ \\
Male gender & $1,239(60.2)$ & $132(71.2)$ & $1,107(59.0)$ & $<0.001$ \\
Diabetes & $922(44.8)$ & $92(50.0)$ & $830(44.3)$ & 0.13 \\
Hypertension & $1,337(64.9)$ & $120(62.2)$ & $1,218(65.0)$ & 0.94 \\
Dyslipidemia & $937(45.5)$ & $77(41.8)$ & $860(45.9)$ & 0.29 \\
Smoking & $385(18.7)$ & $40(21.7)$ & $345(18.4)$ & 0.26 \\
Family history of CAD & $317(15.4)$ & $22(11.9)$ & $295(15.7)$ & 0.17 \\
Chest pain symptoms & $547(26.6)$ & $46(25.0)$ & $501(26.7)$ & 0.61 \\
History of MI & $424(20.6)$ & $63(34.2)$ & $361(19.2)$ & $<0.001$ \\
Dipyridamole stress test & $713(34.6)$ & $95(51.6)$ & $618(33.0)$ & $<0.001$ \\
Rest LVEF (\%) & $55.0 \pm 13.4$ & $50.0 \pm 13.9$ & $55.6 \pm 13.2$ & $<0.001$ \\
Post-stress LVEF (\%) & $57.7 \pm 12.0$ & $51.3 \pm 12.3$ & $58.3 \pm 11.8$ & $<0.001$ \\
Post-stress LVEF $\leq 45 \%$ & $319(15.5)$ & $47(25.5)$ & $272(14.5)$ & $<0.001$ \\
SSS & $3.4 \pm 6.4$ & $6.0 \pm 8.0$ & $3.2 \pm 6.2$ & $<0.001$ \\
SRS & $2.0 \pm 4.8$ & $3.6 \pm 6.4$ & $1.9 \pm 4.6$ & $<0.001$ \\
SDS & $1.4 \pm 2.9$ & $2.3 \pm 3.5$ & $1.3 \pm 2.9$ & $<0.001$ \\
\hline
\end{tabular}

Data given as mean $\pm \mathrm{SD}$ or $\mathrm{n}(\%)$. CAD, coronary artery disease; $\mathrm{LVEF}$, left ventricular ejection fraction; MI, myocardial infarction; SDS, summed difference score; SRS, summed rest score; SSS, summed stress score.

\begin{tabular}{|lccc|}
\hline Table 2. Subject Characteristics vs. Coronary Revascularization & $\begin{array}{c}\text { During Follow-up } \\
\text { Revascularization } \\
(\mathbf{n = 2 0 8 )}\end{array}$ & $\begin{array}{c}\text { No revascularization } \\
\mathbf{( n = 1 , 8 5 1 )}\end{array}$ & P-value \\
Age (years) & $62.5 \pm 8.5$ & $60.8 \pm 10.7$ & 0.02 \\
Male gender & $169(81.2)$ & $1,070(57.8)$ & $<0.001$ \\
Diabetes & $156(75.0)$ & $766(41.4)$ & $<0.001$ \\
Hypertension & $154(74.0)$ & $1,184(64.0)$ & 0.004 \\
Dyslipidemia & $126(60.6)$ & $811(43.8)$ & $<0.001$ \\
Smoking & $75(36.1)$ & $310(16.7)$ & $<0.001$ \\
Family history of CAD & $22(10.6)$ & $295(15.9)$ & $<0.001$ \\
Chest pain symptoms & $65(31.2)$ & $482(26.0)$ & 0.11 \\
History of MI & $90(43.3)$ & $334(18.0)$ & $<0.001$ \\
Dipyridamole stress test & $72(34.6)$ & $641(34.6)$ & 0.99 \\
Rest LVEF (\%) & $53.2 \pm 12.4$ & $55.4 \pm 13.5$ & 0.05 \\
Post-stress LVEF $(\%)$ & $52.6 \pm 12.8$ & $58.2 \pm 11.8$ & $<0.001$ \\
Post-stress LVEF $\leq 45 \%$ & $50(24.0)$ & $269(14.5)$ & $<0.001$ \\
SSS & $9.8 \pm 8.6$ & $2.7 \pm 5.7$ & $<0.001$ \\
SRS & $5.1 \pm 6.3$ & $1.7 \pm 4.5$ & $<0.001$ \\
SDS & $4.7 \pm 5.2$ & $1.0 \pm 2.3$ & $<0.001$ \\
\hline
\end{tabular}

Data given as mean \pm SD or $n(\%)$. Abbreviations as in Table 1 .

martingale residuals. Collinearity was checked using the variance inflation factor. ${ }^{16}$ Finally, the Stata margins command was used to estimate and interpret adjusted predictions and marginal effects of revascularization on hazard ratio. ${ }^{17}$ All statistical analysis was performed with Stata 14.0 (StataCorp, College Station, TX, USA).

\section{Results}

\section{Patient Characteristics and Outcome}

Of the 2,157 patients enrolled, follow-up data were not available for 98 patients $(4.5 \%)$. The mean age of patients lost to follow-up was $59.3 \pm 11.9$ years and the prevalence of cardiovascular risk factors, presenting symptoms, and history of
CAD were similar to those of the 2,059 patients with available follow-up data. Indication for stress MPS was suspected CAD in 1,474 (72\%) and risk stratification of known CAD (ie, history of ACS or coronary revascularization and angiographic evidence of $>50 \%$ diameter coronary stenosis) in $585(28 \%)$. The stressor used (exercise, $n=1,346$; dipyridamole, $n=713$ ) was chosen on the basis of specific contraindications and physician preference. The median follow-up was 61 months (IQR, 46-78 months). During follow-up, 184 endpoint events occurred (8.9\% cumulative event rate). The events were cardiac death in 126 patients and non-fatal MI in 58 patients. Thirty-two patients died of non-cardiac death and were censored at the time of death.

Clinical characteristics and MPS results with and without 
Table 3. Univariate Indicators of Cardiac Death or Non-Fatal MI

$\begin{array}{lccc} & \text { HR (95\% Cl) } & \text { Likelihood ratio } \mathbf{X}^{2} & \text { P-value } \\ \text { Age (per year) } & 1.06(1.04-1.07) & 58.97 & <0.001 \\ \text { Male gender } & 1.82(1.29-2.50) & 13.81 & <0.001 \\ \text { Diabetes } & 1.79(1.33-2.42) & 14.43 & <0.001 \\ \text { Hypertension } & 1.04(0.77-1.41) & 0.06 & 0.79 \\ \text { Dyslipidemia } & 0.91(0.68-1.22) & 0.41 & 0.52 \\ \text { Smoking } & 1.57(1.09-2.23) & 5.69 & 0.02 \\ \text { Family history of CAD } & 0.96(0.61-1.50) & 0.03 & 0.86 \\ \text { Chest pain symptoms } & 1.05(0.75-1.47) & 0.08 & 0.78 \\ \text { History of MI } & 2.72(2.00-3.71) & 35.63 & <0.001 \\ \text { Dipyridamole stress test } & 2.09(1.56-2.79) & 24.45 & <0.001 \\ \text { Post-stress LVEF (per 1\%) } & 0.95(0.94-0.96) & 66.11 & <0.001 \\ \text { SSS (per unit) } & 1.06(1.04-1.08) & 38.43 & <0.001 \\ \text { SRS (per unit) } & 1.06(1.04-1.08) & 25.55 & <0.001 \\ \text { SDS (per unit) } & 2.33(1.72-3.14) & 23.40 & <0.001 \\ \text { Revascularization } & 0.49(0.23-1.01) & 4.66 & 0.03\end{array}$

Abbreviations as in Table 1.
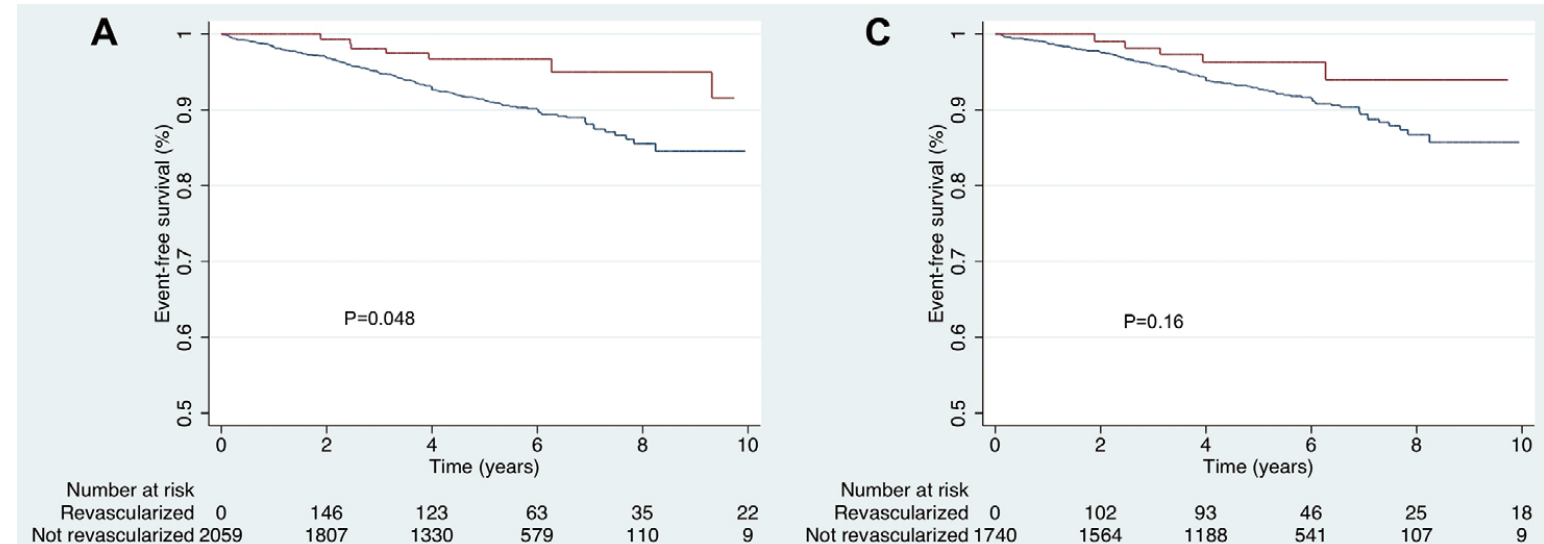

B

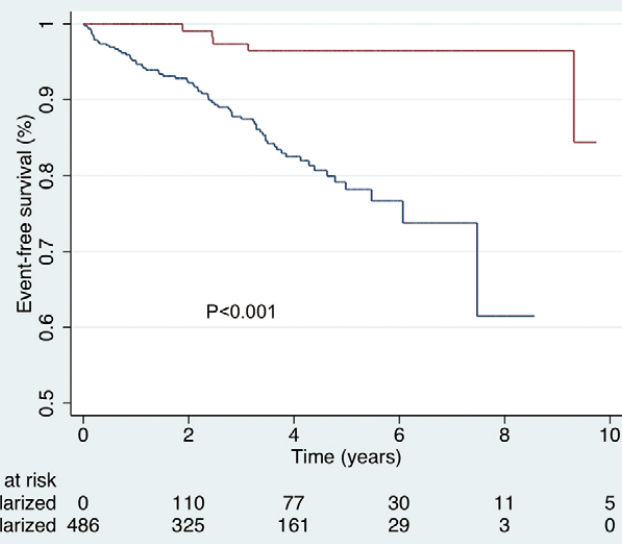

D

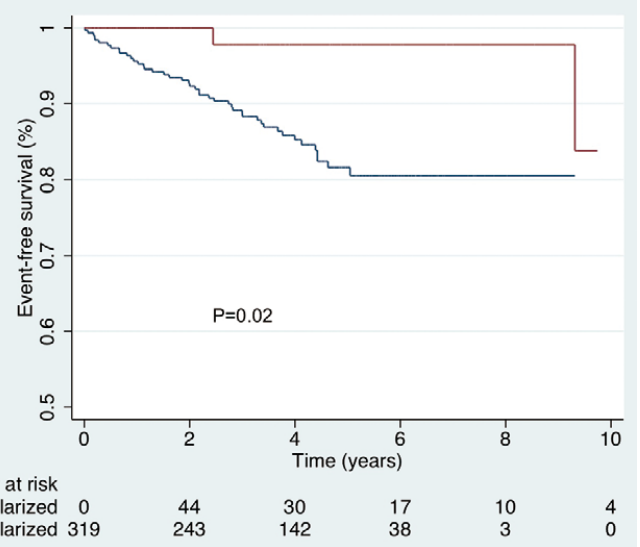

Figure 1. Estimated event-free survival curves for revascularization status (time dependent covariate) in (A) the whole group, (B) patients with stress-induced ischemia, (C) patients with post-stress left ventricular ejection fraction (LVEF) $>45 \%$, and (D) in those with LVEF $\leq 45 \%$. (red line) Revascularized; (blue line) not revascularized. The number at risk in the revascularized group is 0 at the beginning of follow-up (the time of index myocardial perfusion single-photon emission computed tomography) because no patient was in the revascularized group at that time. Thereafter, the study cohorts are continually updated as follows: for the time prior to revascularization, the patient contributes to the no revascularization cohort, but for the time after undergoing revascularization, the patient contributes to the revascularization cohort. 
endpoint events are reported in Table 1 . The mean number of CAD risk factors was similar in patients with and without events (2.6 \pm 1.4 vs. $2.5 \pm 1.4 ; \mathrm{P}=0.19)$. Post-stress $\mathrm{LVEF}$ was $\leq 45 \%$ in 47 patients $(25.5 \%)$ with, and in $272(14.5 \%)$ without events $(\mathrm{P}<0.001)$. Among patients with events, $76(41.3 \%)$ had stress-induced ischemia compared with 410 (21.9\%) without events $(\mathrm{P}=0.001)$. Stress-induced ischemia was mild in 54 and moderate-severe in 22 patients with events, and mild in 292 and moderate-severe in 118 patients without events $(\mathrm{P}<0.001)$. A significant correlation (all $\mathrm{P}<0.001)$ between post-stress LVEF and SSS ( $\mathrm{r}=-0.50), \mathrm{SRS}(\mathrm{r}=-0.48)$ and SDS $(\mathrm{r}=-0.31)$ was found. On multivariate analysis, other significant predictors of reduced post-stress $\mathrm{LVEF}$ were male gender $(\mathrm{P}<0.001)$, diabetes $(\mathrm{P}=0.005)$, dyslipidemia $(\mathrm{P}<0.001)$, prior $\mathrm{MI}(\mathrm{P}=0.002)$ and angina symptoms $(\mathrm{P}=0.01)$.

During follow-up, 208 patients underwent coronary revascularization (coronary artery bypass grafting [CABG], $\mathrm{n}=62$; percutaneous coronary intervention [PCI], $n=146$ ). The median time from index MPS to revascularization was 10.2 months (IQR, 3.7-22.6 months). Clinical characteristics and MPS results according to presence of revascularization are listed in Table 2. Post-stress LVEF was $\leq 45 \%$ in 50 patients $(24 \%)$ with, and in $269(14.5 \%)$ without revascularization $(\mathrm{P}<0.001)$. Among patients with revascularization, $130(62.5 \%)$ had stress-induced ischemia compared with 356 (19.2\%) without revascularization $(\mathrm{P}<0.001)$. Stress-induced ischemia was mild in 74 and moderate-severe in 56 patients with revascularization, and mild in 272 and moderate-severe in 84 patients without revascularization $(\mathrm{P}<0.001)$. The cumulative event rate was $3.8 \%$ in patients with revascularization and $9.5 \%$ in those without revascularization $(\mathrm{P}=0.007)$. The event rate was similar in patients undergoing CABG and those treated with PCI. When patients were classified into 4 subsets according to occurrence of events and revascularization, the clinical characteristics and MPS findings were significantly different (all $\mathrm{P}<0.001$ ) across the groups, but chest pain symptoms were similar (Table S1).

\section{Univariate Predictors of Cardiac Death and Non-Fatal MI}

Univariate associations of clinical data, stress MPS results and

\begin{tabular}{|lcc|}
\hline \multicolumn{3}{|c|}{ Table 4. Multivariate Indicators of Cardiac Death or } \\
Non-Fatal MI & HR (95\% Cl) & P-value \\
Age (per year) & $1.05(1.03-1.07)$ & $<0.001$ \\
Male gender & $1.37(0.98-1.92)$ & 0.07 \\
History of MI & $1.56(1.09-2.18)$ & 0.015 \\
Dipyridamole stress test & $1.81(1.34-2.43)$ & $<0.001$ \\
Post-stress LVEF (per 1\%) & $0.96(0.94-0.97)$ & $<0.001$ \\
SDS (per unit) & $1.06(1.02-1.11)$ & 0.005 \\
Revascularization & $0.19(0.09-0.42)$ & $<0.001$ \\
Revascularization×SDS & $0.88(0.78-0.99)$ & 0.045 \\
Post-stress LVEF×SDS & $1.01(1.01-1.02)$ & 0.015 \\
\hline
\end{tabular}

Abbreviations as in Table 1.

revascularization with cardiac death or non-fatal MI are given in Table 3. Event-free survival was slightly better in patients with revascularization than in those without (Mantel-Byar $\chi^{2}$, 3.91; $\mathrm{P}=0.048$; Figure 1A). When only patients with evidence of stress-induced ischemia ( $\mathrm{SDS} \geq 2$ ) were considered, the survival benefit of revascularization was greater (Mantel-Byar $\chi^{2}, 14.85$; $\mathrm{P}<0.001$; Figure 1B). Conversely, no difference in event-free survival was seen when only patients without stress-induced ischemia $(\mathrm{n}=1,573)$ were considered (MantelByar $\chi^{2}, 1.16 ; \mathrm{P}=0.28$ ). When patients were stratified according to post-stress LVEF, survival benefit was seen in those with $\mathrm{LVEF} \leq 45 \%$ (Mantel-Byar $\chi^{2}, 4.82 ; \mathrm{P}=0.02$ ) but not in those with LVEF $>45 \%$ (Mantel-Byar $\chi^{2}, 1.97$; $\mathrm{P}=0.16$; Figures 1C,D).

\section{Multivariate Modeling}

The results of Cox multivariate analysis for prediction of cardiac death or non-fatal MI are given in Table 4. SDS, poststress LVEF and revascularization were associated with outcome. Among the interactions tested, A significant interaction was seen between revascularization and SDS $(\mathrm{P}=0.045)$ and between post-stress LVEF and SDS $(\mathrm{P}=0.015)$. The final

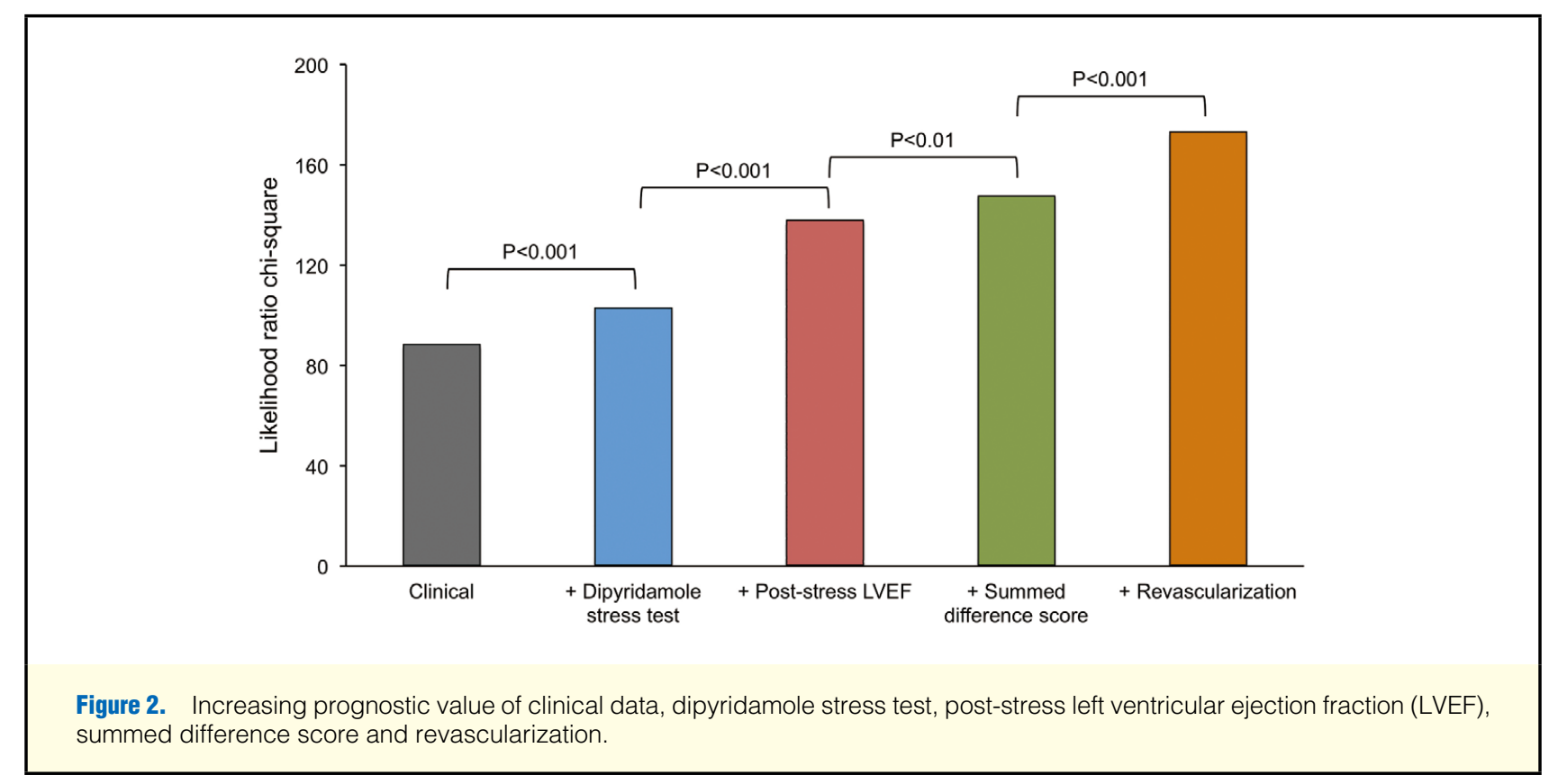



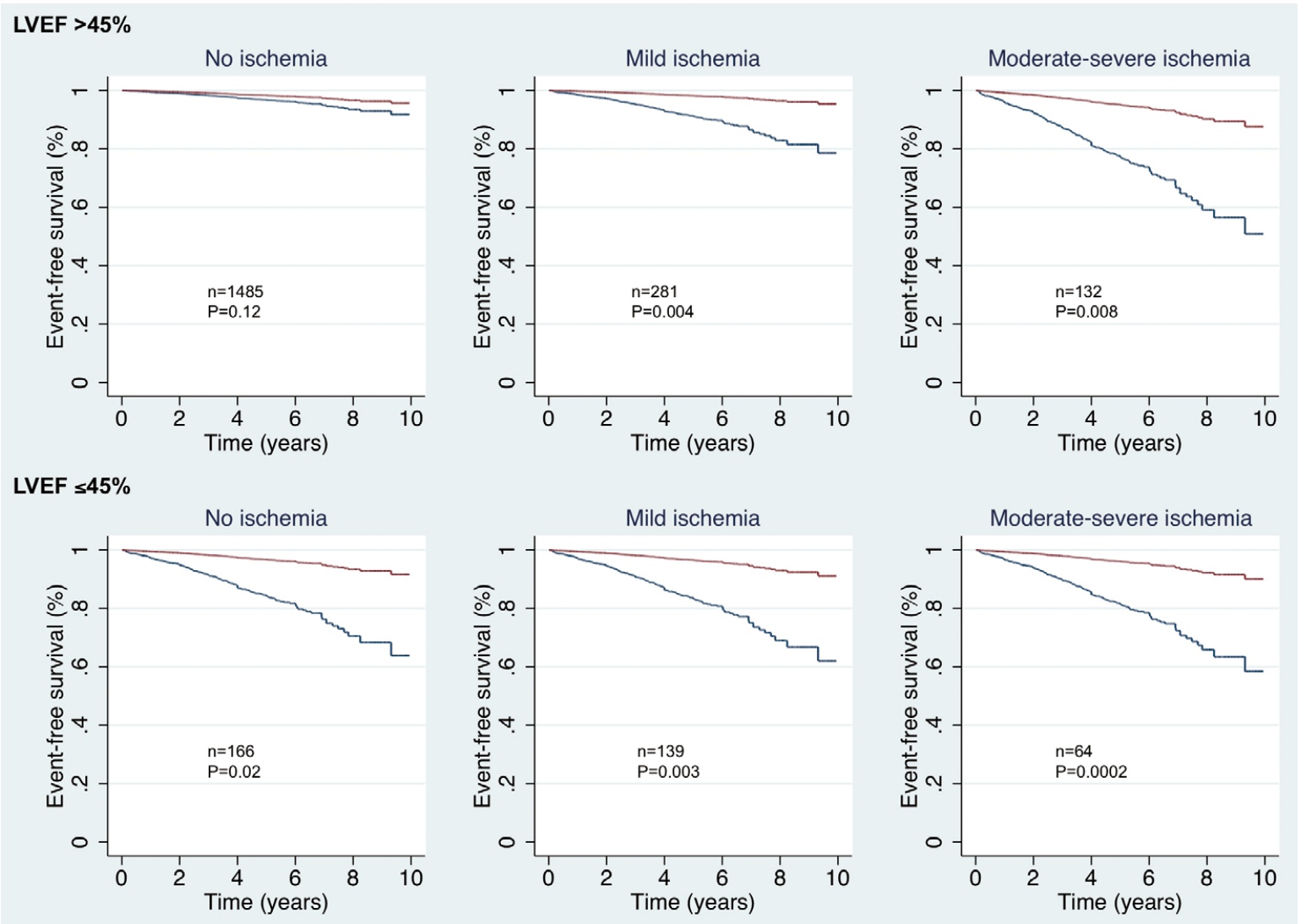

Figure 3. Event-free survival curves estimated using Cox modeling as function of the degree of stress-induced ischemia, poststress left ventricular ejection fraction (LVEF) and revascularization status (time-dependent covariate), at means of the other covariates included in the final multivariate Cox model. (red line) Revascularized; (blue line) not revascularized. In patients with normal post-stress LVEF, revascularization rate and event rate (person/years) were $4.7 \%$ and $1.2 \%$ in those without ischemia, $22.2 \%$ and $3.3 \%$ in those with mild ischemia, and $43.5 \%$ and $3.5 \%$ in those with moderate-severe ischemia. In patients with reduced post-stress LVEF, revascularization rate and event rate (person/years) were $7.1 \%$ and $2.4 \%$ in those without ischemia, $19.8 \%$ and $4.3 \%$ in those with mild ischemia, and $33.3 \%$ and $4.8 \%$ in those with moderate-severe ischemia.

model including these interactions was strongly associated with the occurrence of endpoint events (likelihood ratio $\chi^{2}$, 176.68; $\mathrm{P}<0.001)$. The optimal SDS cut-off for effective revascularization was $\geq 6\left(\log \operatorname{rank} \chi^{2}, 8.35 ; \mathrm{P}=0.004\right)$ in patients with normal post-stress LVEF and $\geq 3$ (log rank $\chi^{2}$, 7.37; $\mathrm{P}=0.007$ ) in those with reduced post-stress LVEF. The increased value of significant predictors identified on multivariate analysis is shown in Figure 2. A separate analysis was performed in patients without and with reduced post-stress LVEF (Table S2). In patients with normal post-stress LVEF, age, male gender, prior MI, dipyridamole stress test and SDS were all significant predictors of events, while in those with reduced post-stress LVEF, prior MI and dipyridamole stress test were significantly associated with events.

Estimated event-free survival curves based on the Cox model for patients without ischemia, mild ischemia and moderate-severe ischemia according to post-stress LVEF are reported in Figure 3. In patients without ischemia, no difference was seen in estimated survival in the revascularization and no revascularization groups in the presence of normal LVEF, whereas in the presence of reduced LVEF, revascularization had a beneficial effect on event-free survival. The marginal effect of revascularization on HR at different levels of SDS and post-stress LVEF is shown in Figure 4. In patients with preserved LVEF the reduction of HR induced by revascularization increases as SDS increases. For SDS $<6$, however, the reduction in HR was statistically significant only in the presence of reduced LVEF. In contrast, the beneficial effect of revascularization was smaller in the presence of reduced LVEF, and was largely independent of SDS.

\section{Discussion}

We assessed in a large series of patients with known or suspected CAD the impact of gated stress MPS data on survival benefit after coronary revascularization during long-term follow-up. Unadjusted analysis showed an evident survival benefit from revascularization in patients with stress-induced ischemia. When patients were stratified according to poststress LVEF, on unadjusted analysis a survival benefit was seen only in those with LVEF $\leq 45 \%$.

On multivariate Cox modeling the protective effect of revascularization increased as SDS increased, in particular for preserved LVEF. Of note, in the absence of stress-induced ischemia, the survival benefit of revascularization was seen only for $\mathrm{LVEF} \leq 45 \%$. In the presence of moderate-severe 


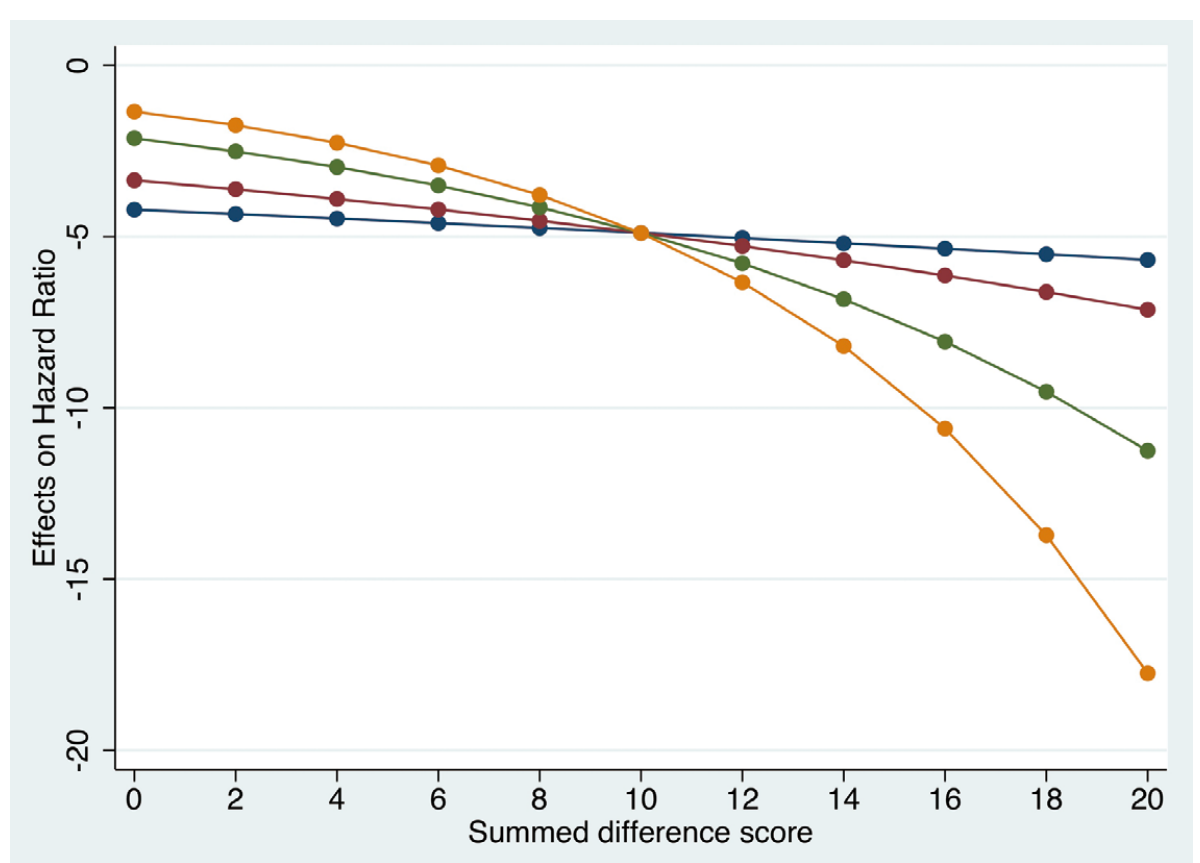

Figure 4. Conditional marginal effects of coronary revascularization on hazard ratio (HR) estimated on Cox modeling according to stress-induced ischemia and post-stress left ventricular ejection fraction (EF: blue, 30\%; maroon, 40\%; green, 50\%; orange, $60 \%$ ), and at means of the other covariates included in the Cox model. For summed difference score (SDS) $<6$ the reduction in $\mathrm{HR}$ induced by revascularization is statistically significant only for reduced EF. The reduction in HR is largely independent of SDS when EF is reduced. Conversely, in the presence of normal EF the reduction in HR increases as SDS increases, and the relationship becomes steeper for SDS $\geq 10$.

stress-induced ischemia, the survival benefit was greater for LVEF $>45 \%$ (Figure 3). Due to the significant interaction between SDS and post-stress LVEF, for SDS $<6$ the reduction in HR was statistically significant only in the presence of reduced LVEF. Conversely, the protective effect of coronary revascularization was greater in patients with severe ischemia and preserved LVEF (Figure 4).

\section{Previous Studies}

The role of cardiac nuclear medicine in identifying patients that would benefit from coronary revascularization has been previously investigated. ${ }^{18-20}$ Hachamovitch et al found that, during short-term follow-up ( $1.9 \pm 0.6$ years), early revascularization reduced the absolute and relative risk of cardiac death more than medical therapy in patients with moderate-large inducible ischemia on stress MPS and no prior MI or revascularization. ${ }^{3}$ In a subsequent study, the same group of researchers addressed the value of combining post-stress LVEF and myocardial ischemia data to gather information on survival benefit after early revascularization. ${ }^{5}$ As in the present study, those authors found that post-stress LVEF and the degree of inducible myocardial ischemia were independent predictors of future cardiac events. They found that, however, for any degree of MPS ischemia, the therapeutic benefit derived from subsequent myocardial revascularization was greater in patients with reduced LVEF, ${ }^{5}$ while in the present study the therapeutic benefit of revascularization was higher in the presence of preserved LVEF. Differences in subjects and in study design might explain the different results observed. In the present study, the prevalence of diabetes was higher and more patients had previous MI. We found a significant correlation between post-stress LVEF and SRS, which was 7.1 \pm 8.2 and $1.1 \pm 3.1$ in patients with post-stress $\mathrm{LVEF} \leq 45 \%$ and $>45 \%$, respectively $(\mathrm{P}<0.001)$. It has been reported that the amount of ischemic myocardium predicts survival benefit of early revascularization in patients without prior MI, whereas no such benefit was present in patients with prior MI and evidence of extensive scar, in agreement with the attenuated survival benefit from coronary revascularization observed in patients with severe ischemia and low post-stress LVEF. ${ }^{3}$ It should also be noted that a 60- or a 90-day window for therapy selection assignment to mimic a randomized clinical trial has been used, based on the assumption that revascularizations performed within this time frame are based mainly on the results of MPS rather than on clinical status..$^{3-5,21}$ The potential effects of revascularization performed later after index MPS, however, have not been analyzed and this approach may bias the results observed. The biases involved in comparing timeto-event data for different groups when the group membership varies during the study have been recognized. ${ }^{15,22,23}$ In the present study, we used an alternative approach, and treated the first revascularization after the index MPS as a binary timedependent covariate. With this approach, the patient's treatment category is allowed to change at the time of revascularization, and the time before revascularization is counted as medical therapy time and subsequent time as revascularization time. ${ }^{24}$

The present findings are in agreement with the results of the Surgical Treatment for IsChemic Heart Failure (STICH) trial. ${ }^{25}$ In that study there was a significant benefit in terms of death or cardiovascular hospitalization for revascularization 
compared with medical therapy in patients with CAD and severe LV dysfunction. The presence of inducible ischemia, however, did not identify those with greater therapeutic benefit and no interaction was found between revascularization and ischemia. These data support the concept that the therapeutic effect of revascularization is not limited to patients with inducible ischemia on stress testing.

\section{Study Limitations}

The present study was a single-center cohort study and carries all of the inherent limitations of that study design. Further, the decision to submit a patient to revascularization was made by the referring physician and therefore some patients with moderate-severe ischemia did not undergo revascularization. The lack of randomization to evaluate the impact of revascularization on outcome represents a major limitation and no definitive treatment benefit may be inferred by the present exploratory analysis. Two randomized clinical trials, the Clinical Outcomes Utilizing Revascularization and Aggressive Drug Evaluation (COURAGE) and Bypass Angioplasty Revascularization Investigation 2 Diabetes (BARI 2D), reported that the impact of aggressively applied medical therapy on patient survival is not further improved by the addition of PCI. ${ }^{26,27}$ Substudies of both trials, however, showed greater reduction in MPS ischemia after revascularization, although a morbidity and mortality benefit was not observed. ${ }^{28,29}$ A subsequent substudy from COURAGE indicates that the extent of ischemia did not predict adverse events and did not alter treatment effectiveness. ${ }^{30}$ It has been suggested that a potential explanation for failure of revascularization to reduce the incidence of death or MI in these trials is that low-risk patients were enrolled, reducing the power to show a benefit from revascularization. Thus, the effect of revascularization as compared with medical therapy in patients with moderate-severe ischemia is still under investigation in the International Study of Comparative Health Effectiveness with Medical and Invasive Approaches (ISCHEMIA) trial. ${ }^{31} \mathrm{We}$ assumed that all patients were treated appropriately during follow-up. Whether the use of maximum medical therapy would yield the same results is beyond the scope of the present study. Another limitation is the lack of detailed anatomic data as well as information on the completeness of revascularization, which would assist in the interpretation of the findings. Finally, no comparison of CABG and PCI was made because of insufficient power. Given that both procedures potentially reduce the amount of inducible ischemia, we believe that combining these 2 procedures does not compromise the present results.

\section{Conclusions}

On long-term follow-up, both the degree of stress-induced myocardial ischemia and post-stress LVEF should be taken into account for predicting the effect of revascularization on outcome in patients with suspected or known CAD. In patients with reduced LVEF the revascularization-induced reduction in $\mathrm{HR}$ is largely independent of stress-induced ischemia. Conversely, in those patients with preserved LVEF the protective effect of revascularization progressively increases as SDS increases.

\section{Disclosures}

The authors declare no conflicts of interest.

\section{References}

1. Shaw LJ, Hage FG, Berman DS, Hachamovitch R, Iskandrian A. Prognosis in the era of comparative effectiveness research: Where is nuclear cardiology now and where should it be? J Nucl Cardiol 2012; 19: 1026-1043.

2. Cortigiani L, Borelli L, Raciti M, Bovenzi F, Picano E, Molinaro S, et al. Prediction of mortality by stress echocardiography in 2835 diabetic and 11305 nondiabetic patients. Circ Cardiovasc Imaging 2015; 8: e002757, doi:10.1161/CIRCIMAGING.114.002757.

3. Hachamovitch R, Rozanski A, Shaw LJ, Stone GW, Thomson LE, Friedman JD, et al. Impact of ischaemia and scar on the therapeutic benefit derived from myocardial revascularization vs. medical therapy among patients undergoing stress-rest myocardial perfusion scintigraphy. Eur Heart J 2011; 32: 1012-1024.

4. Hachamovitch R, Hayes SW, Friedman JD, Cohen I, Berman DS. Comparison of the short-term survival benefit associated with revascularization compared with medical therapy in patients with no prior coronary artery disease undergoing stress myocardial perfusion single photon emission computed tomography. Circulation 2003; 107: 2900-2907.

5. Hachamovitch R, Rozanski A, Hayes SW, Thomson LE, Germano G, Friedman JD, et al. Predicting therapeutic benefit from myocardial revascularization procedures: Are measurements of both resting left ventricular ejection fraction and stress-induced myocardial ischemia necessary? J Nucl Cardiol 2006; 13: 768-778.

6. Hesse B, Tägil K, Cuocolo A, Anagnostopoulos C, Bardiés M, Bax $\mathrm{J}$, et al; EANM/ESC Group. EANM/ESC procedural guidelines for myocardial perfusion imaging in nuclear cardiology. Eur J Nucl Med Mol Imaging 2005; 32: 855-897.

7. Petretta M, Acampa W, Evangelista L, Daniele S, Ferro A, Cuocolo A; Impact of Inducible Ischemia by Stress SPECT (IDIS) Investigators. Impact of inducible ischemia by stress SPECT in cardiac risk assessment in diabetic patients: Rationale and design of a prospective, multicenter trial. J Nucl Cardiol 2008; 15: 100-104.

8. Acampa W, Petretta M, Evangelista L, Daniele S, Xhoxhi E, De Rimini ML, et al. Myocardial perfusion imaging and risk classification for coronary heart disease in diabetic patients: The IDIS study: A prospective, multicentre trial. Eur J Nucl Med Mol Imaging 2012; 39: $387-395$.

9. Germano G, Kavanagh PB, Waechter P, Areeda J, Van Kriekinge S, Sharir T, et al. A new algorithm for the quantitation of myocardial perfusion SPECT. I: Technical principles and reproducibility. J Nucl Med 2000; 41: 712-719.

10. Sharir T, Germano G, Kang X, Lewin HC, Miranda R, Cohen I, et al. Prediction of myocardial infarction versus cardiac death by gated myocardial perfusion SPECT: Risk stratification by the amount of stress-induced ischemia and the post-stress ejection fraction. $\mathrm{J} \mathrm{Nucl}$ Med 2001; 42: 831-837.

11. Acampa W, Petretta M, Daniele S, Del Prete G, Assante R, Zampella $\mathrm{E}$, et al. Incremental prognostic value of stress myocardial perfusion imaging in asymptomatic diabetic patients. Atherosclerosis 2013; 227: $307-312$

12. Sharir T, Germano G, Kavanagh PB, Lai S, Cohen I, Lewin HC, et al. Incremental prognostic value of post-stress left ventricular ejection fraction and volume by gated myocardial perfusion single photon emission computed tomography. Circulation 1999; 100: 10351042.

13. Thygesen K, Alpert JS, Jaffe AS, Simoons ML, Chaitman BR, White HD; Joint ESC/ACCF/AHA/WHF Task Force for the Universal Definition of Myocardial Infarction. Third universal definition of myocardial infarction. Circulation 2012; 126: 2020-2035.

14. Simon R, Makuch RW. A non-parametric graphical representation of the relationship between survival and the occurrence of an event: Application to responder versus non-responder bias. Stat Med 1984; 3: $35-44$.

15. Mantel N, Byar DP. Evaluation of response time data involving transient states: An illustration using heart-transplantation data. J Am Stat Assoc 1974; 69: 81-86.

16. Vittinghoff E, Glidden D, Shiboski SC, McCulloch CE. Regression methods in biostatistics: Linear, logistic, survival, and repeated measures models, 2nd edn. New York, NY: Springer, 2012.

17. Buis ML. Stata tip 87: Interpretation of interactions in nonlinear models. Stata J 2010; 10: 305-308.

18. Naya M, Tamaki N, Tsutsui H. Coronary flow reserve estimated by positron emission tomography to diagnose significant coronary artery disease and predict cardiac events. Circ J 2015; 79: 15-23.

19. Furuhashi T, Moroi M, Awaya T, Minakawa M, Masai H, Kunimasa $\mathrm{T}$, et al. Usefulness of stress myocardial perfusion imaging and baseline clinical factors for predicting cardiovascular events in patients with prior coronary artery disease. Circ J 2014; 78: 16761683.

20. Nishiyama Y, Miyagawa M, Kawaguchi N, Nakamura M, Kido T, Kurata A, et al. Combined supine and prone myocardial perfusion 
single-photon emission computed tomography with a cadmium zinc telluride camera for detection of coronary artery disease. Circ $J$ 2014; 78: 1169-1175.

21. Staniloff HM, Forrester JS, Berman DS, Swan HJ. Prediction of death, myocardial infarction, and worsening chest pain using thallium scintigraphy and exercise electrocardiography. J Nucl Med 1986; 27: $1842-1848$.

22. Gail MH. Does cardiac transplantation prolong life?: A reassessment. Ann Intern Med 1972; 76: 815-817.

23. Clark DA, Stinson EB, Griepp RB, Schroeder JS, Shumway NE, Harrison DC. Cardiac transplantation in man, VI: Prognosis of patients selected for cardiac transplantation. Ann Intern Med 1971; 75: $15-21$.

24. Oakes D, Peterson DR. Survival methods: Additional topics. Circulation 2008; 117: 2949-2955.

25. Panza JA, Holly TA, Asch FM, She L, Pellikka PA, Velazquez EJ, et al. Inducible myocardial ischemia and outcomes in patients with coronary artery disease and left ventricular dysfunction. J Am Coll Cardiol 2013; 61: 1860-1870.

26. Boden WE, O'Rourke RA, Teo KK, Hartigan PM, Maron DJ, Kostuk WJ, et al; COURAGE Trial Research Group. Optimal medical therapy with or without PCI for stable coronary disease. $N$ Engl $J$ Med 2007; 356: 1503-1516.

27. BARI 2D Study Group, Frye RL, August P, Brooks MM, Hardison RM, Kelsey SF, MacGregor JM, et al. A randomized trial of therapies for type 2 diabetes and coronary artery disease. $N$ Engl $J$ Med 2009; 360: 2503-2515.

28. Shaw LJ, Berman DS, Maron DJ, Mancini GB, Hayes SW, Hartigan PM, et al; COURAGE Investigators. Optimal medical therapy with or without percutaneous coronary intervention to reduce ischemic burden: Results from the Clinical Outcomes Utilizing Revascularization and Aggressive Drug Evaluation (COURAGE) trial nuclear substudy. Circulation 2008; 117: 1283-1291.

29. Shaw LJ, Cerqueira MD, Brooks MM, Althouse AD, Sansing VV, Beller GA, et al. Impact of left ventricular function and the extent of ischemia and scar by stress myocardial perfusion imaging on prognosis and therapeutic risk reduction in diabetic patients with coronary artery disease: Results from the Bypass Angioplasty Revascularization Investigation 2 Diabetes (BARI 2D) trial. J Nucl Cardiol 2012; 19: $658-669$.

30. Shaw LJ, Weintraub WS, Maron DJ, Hartigan PM, Hachamovitch $\mathrm{R}$, Min JK, et al. Baseline stress myocardial perfusion imaging results and outcomes in patients with stable ischemic heart disease randomized to optimal medical therapy with or without percutaneous coronary intervention. Am Heart J 2012; 164: 243-250.

31. Gibbons RJ, Miller TD. Should extensive myocardial ischaemia prompt revascularization to improve outcomes in chronic coronary artery disease? Eur Heart J 2015; 36: 2281-2287.

\section{Supplementary Files}

Supplementary File 1

Table S1. Subject characteristics vs. cardiac death or non-fatal MI and coronary revascularization during follow-up

Table S2. Multivariate indicators of cardiac death or non-fatal MI vs. reduced post-stress LVEF

Please find supplementary file(s);

http://dx.doi.org/10.1253/circj.CJ-15-1093 